\title{
Dynamics of Argon Clusters in an Intense Laser Pulse: Bloch-Like Hydrodynamic Model
}

\author{
M. Rusek And A. OrŁowski \\ Institute of Physics, Polish Academy of Sciences \\ al. Lotników 32/46, 02-668 Warsaw, Poland
}

(Received May 7, 2004)

\begin{abstract}
The dynamics of small ( $\leq 55$ atoms) argon clusters ionized by an intense, infrared, femtosecond laser pulse is studied using a Bloch-like hydrodynamic model. Evolution of both free electrons and ions formed in the cluster explosion process is examined. Oscillations of the electron cloud in a rare-gas atomic cluster are described as a motion of a fluid obeying Bloch-like hydrodynamic equations. Our theoretical approach includes all possible ionization mechanisms: tunnel (or field) ionization both by an external laser field, and by an internal field due to the space-charge distribution inside the cluster, as well as electron-impact (or collisional) ionization. The results of our simulations are compared both with experimental findings and with predictions of other theoretical models.
\end{abstract}

PACS numbers: 36.40.--c, 32.80.Rm, 42.50.Hz, 07.05.Tp

\section{Introduction}

Atomic clusters formed in expanding high-pressure gas jet may be considered as targets of intermediate size between gaseous media and solids. They are as easily penetrated by a laser beam as gaseous media and at the same time exhibit large absorption of laser energy comparable to the solid targets [1].

Laser interaction with the atomic clusters (consisting of a few hundred to few thousand atoms) differs substantially from that of simple atomic and molecular systems. Experiments on clusters irradiated by the intense laser pulses have revealed efficient generation of extremely highly charged atomic ions $[2-8]$ and generation of electrons and ions with $\mathrm{MeV}$ kinetic energies [6, 7, 9-11]. Several other theoretical models have been proposed to explain the mechanism underlying the production of highly charged energetic ions in interaction of atomic clusters with intense laser pulses. 
In classical Monte Carlo simulations of cluster explosion the nuclei and unbound electrons are treated as classical particles obeying Newton's equations of motion [12-15]. The bound electrons are released with a certain probability depending on the electric field strength inside the cluster [12-15] and collision probability with other electrons and ions [13, 14].

Production of highly charged energetic ions by laser irradiation is of an essentially many-electron character and thus requires a theoretical framework within which a large number of electrons could be treated. The time-dependent density functional theory (TDFT) provides such a framework. Recently an application of this theory to a study of the response of atomic clusters to an intense laser pulse appeared [16]. A simplified one-dimensional model with frozen ion positions and the omission of correlation effects allowed the authors to solve numerically time-dependent Kohn-Sham equations describing electron dynamics in a cluster. Results concerning the initial stage of ionization in moderate intensity regime were presented.

In this paper we further investigate a theoretical approach to explosion of rare-gas atomic clusters in intense laser pulses based on a Bloch-like hydrodynamic model introduced in our previous work [17]. Results of additional simulations are presented and their relation to the conclusions of the recent time-dependent density functional [16] and molecular dynamics models [12-15] is discussed. The accuracy and speed of the Smooth Particle Hydrodynamics N-Body numerical code used in our simulations has been greatly improved. This allows us to study a realistic case of 55 atom argon cluster (in Ref. [17] only a 6 atom argon cluster was studied).

\section{Model}

Following Bloch [18] we assume that the oscillations of the electron cloud in a rare-gas atomic cluster can be viewed as a motion of a fluid characterized by a density $\rho(\boldsymbol{r}, t)$, a velocity field $\boldsymbol{v}(\boldsymbol{r}, t)$, and internal energy $U(\boldsymbol{r}, t)$ obeying the standard conservation equations for number of particles and momentum. In its Lagrangian form, they read as

$$
\begin{aligned}
& \frac{\mathrm{d} \rho(\boldsymbol{r}, t)}{\mathrm{d} t}=-\rho(\boldsymbol{r}, t) \boldsymbol{\nabla} \cdot \boldsymbol{v}(\boldsymbol{r}, t), \\
& m \frac{\mathrm{d} \boldsymbol{v}(\boldsymbol{r}, t)}{\mathrm{d} t}=-\frac{1}{\rho(\boldsymbol{r}, t)} \boldsymbol{\nabla} p(\boldsymbol{r}, t)+e \boldsymbol{\nabla} \Phi(\boldsymbol{r}, t),
\end{aligned}
$$

where the pressure $p$ is defined by the equation of state for an ideal fermion gas [19]:

$$
p(\boldsymbol{r}, t)=\frac{2}{3} \rho(\boldsymbol{r}, t) U(\boldsymbol{r}, t)
$$

and the relations for the internal (kinetic) energy of an ideal electron gas at zero 
temperature [19] are used locally

$$
U(\boldsymbol{r})=c_{k}[\rho(\boldsymbol{r})]^{2 / 3}, \quad c_{k}=\frac{3}{5} \frac{\hbar^{2}}{2 m}\left(3 \pi^{2}\right)^{2 / 3}
$$

( $e$ is the elementary charge, and $m$ denotes the electron mass), and the self-consistent Coulomb potential reads as

$$
\Phi(\boldsymbol{r}, t)=\sum_{a=1}^{N} \frac{Z e}{\left|\boldsymbol{r}-\boldsymbol{R}_{a}(t)\right|}-\int \mathrm{d}^{3} r^{\prime} \frac{e \rho\left(\boldsymbol{r}^{\prime}\right)}{\left|\boldsymbol{r}-\boldsymbol{r}^{\prime}\right|} .
$$

The hydrodynamic equations (1) for the electron density $\rho$ should be supplemented by the Newton equations of motion for the positions of the nuclei $\boldsymbol{R}_{a}$ :

$$
M \frac{\mathrm{d}^{2} \boldsymbol{R}_{a}(t)}{\mathrm{d} t^{2}}=-Z e \boldsymbol{\nabla} \Phi\left(\boldsymbol{R}_{a}(t), t\right),
$$

where $M$ denotes the nuclear mass.

The interaction with the laser pulse is treated within the dipole approximation by replacing the electrostatic Coulomb potential in Eqs. (1) and (5) by

$$
\Phi(\boldsymbol{r}, t) \rightarrow \Phi(\boldsymbol{r}, t)-\boldsymbol{r} \cdot p \mathcal{F}(t),
$$

where $\mathcal{F}(t)$ is the electric field of the incoming wave. The linearly polarized wave of a pulse used in the simulations is assumed to have a field envelope proportional to sine squared with a full width at half maximum $\tau$ and an optical period $\tau_{0}$ :

$$
\mathcal{F}(t)=\mathcal{F}_{0} \sin ^{2}\left(\frac{\pi}{2 \tau} t\right) \cos \left(\frac{2 \pi}{\tau_{0}} t\right) .
$$

\section{Simulations}

The initial structure $\left\{\boldsymbol{R}_{a}\left(t_{0}\right)\right\}$ of the 55 and 13 atom argon clusters is chosen to be that of a closed-shell icosahedron with an atom spacing of $3.7 \AA$ [17]. The initial value of the electron density $\rho\left(\boldsymbol{r}, t_{0}\right)$ is obtained by looking for the stationary solution of the hydrodynamic equations (1) with small viscosity terms added [17]. This initial electron density corresponds to the minimum of the Thomas-Fermi energy functional [17].

The atoms of the cluster have initially no kinetic energy and are subject to an oscillating electric laser field at intensity $I=1.4 \times 10^{15} \mathrm{~W} / \mathrm{cm}^{2}$ (or $\mathcal{F}_{0}=0.2$ a.u.). The pulse used in the simulations had a wavelength $\lambda=800 \mathrm{~nm}$ and a temporal full width at half maximum $\tau=106.67 \mathrm{fs}$ ( or $\tau / \tau_{0}=40$ ).

In Fig. 1 we plot the internal, kinetic, potential, and total energy of a 55 atom argon cluster illuminated by a laser pulse plotted versus time. Total energy absorbed from the laser pulse is clearly visible. Efficient laser energy absorption starts when the pulse envelope approaches its maximal value. It is seen that the Thomas-Fermi energy relations are preserved at the initial time, i.e. the potential energy is twice as large as the internal energy with the minus sign [20]: 


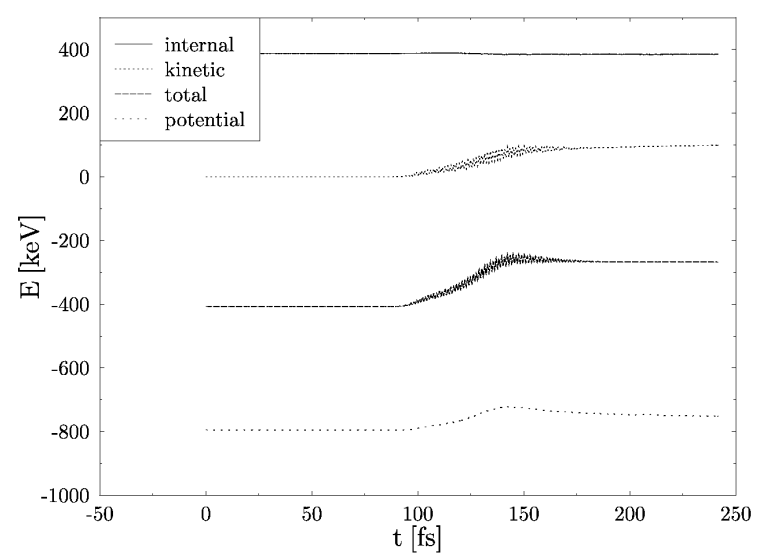

Fig. 1. Internal, kinetic, potential, and total energy of a 55 atom argon cluster illuminated by a laser pulse ploted versus time. The Thomas-Fermi energy relations are preserved at the initial time. Total energy absorbed from the laser pulse is clearly visible. Efficient laser energy absorption starts when the pulse envelope approaches its maximal value.

$$
2 \mathcal{E}_{\text {int }}+\mathcal{E}_{\text {pot }}=0
$$

where

$$
\mathcal{E}_{\text {int }}=\int \mathrm{d}^{3} r \rho(\boldsymbol{r}) U(\boldsymbol{r})
$$

and

$$
\mathcal{E}_{\text {pot }}=\frac{1}{2} \int \mathrm{d}^{3} \operatorname{re\rho }(\boldsymbol{r}) \Phi(\boldsymbol{r})
$$

The total energy absorbed by the cluster from the laser pulse is divided into three parts: kinetic energy of the free (detected) electrons, excitation energy of the ions (in our model it is equal to the kinetic energy of the electrons still bound in the ions), and kinetic energy of the ions. The detailed energy absorption process is illustrated in Fig. 2. First at $\approx 80$ fs the bound electrons are excited into states of high kinetic energy. Thus both ionization of the atoms and expansion of the cluster starts. At later times around $\approx 80$ fs plasmon-like excitations of the free electrons still inside the cluster absorb most of the laser energy. This absorbed energy is then converted into translational energy of the ions and cluster disintegrates by a Coulomb explosion process.

In Fig. 3 we plot the radii $\mathcal{R}$ of the shells of the 55 atom cluster as a function of time $t$. These radii were computed as an average distance between each ion from a given shell and the central ion of the cluster. For convenience the second shell has been split into two subshells with different initial radii (i.e., a subshell is defined as a group of atoms equally spaced from the central atom of the cluster). It is seen that the explosion is neither instantaneous nor uniform. It exhibits a layer-like 


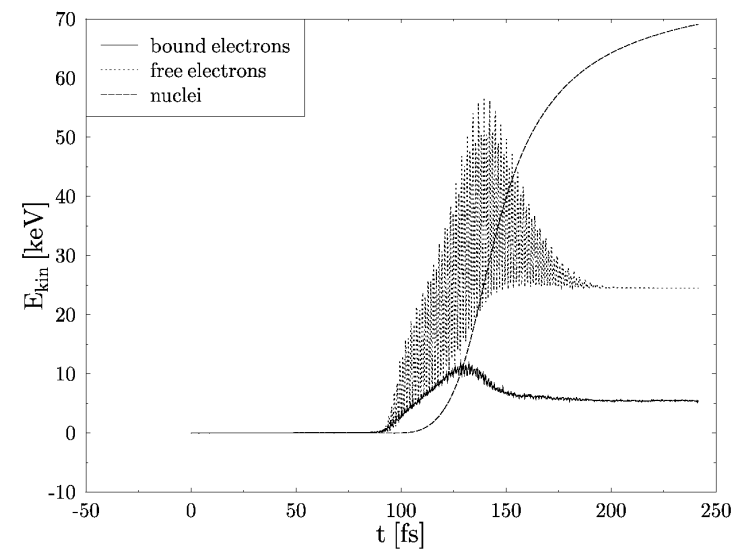

Fig. 2. Kinetic energy of both bound and free (detected) electrons, and the kinetic energy of the ions formed in the process of cluster explosion ploted versus time. Electrons start to move first. Energy acquired by the cluster is next translated into kinetic energy of the ions.

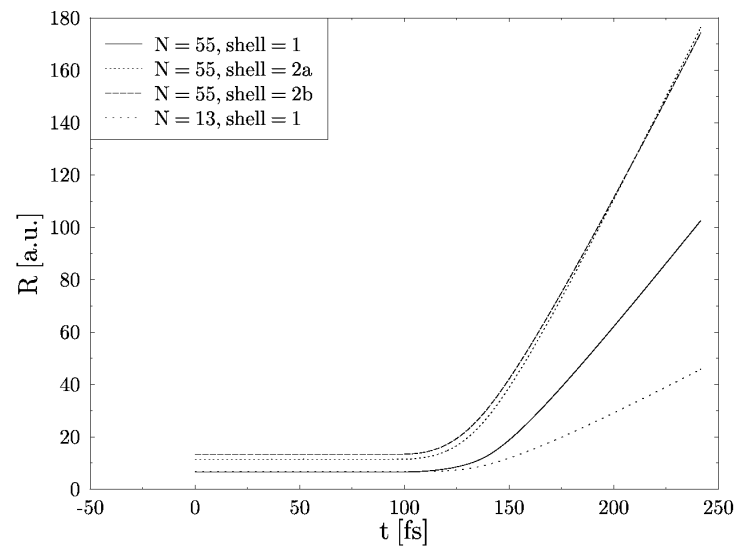

Fig. 3. Radii of each (sub)shell of an exploding 55 atom cluster ploted versus time. The explosion process is neither instantaneous nor uniform. It exhibits a layer-like structure in which shells of cluster ions are expelled sequentially. For comparison a radius of a 13 atom cluster illuminated by the same pulse is also ploted.

structure in which shells of cluster ions are expelled sequentially. For comparison a radius of a 13 atom cluster illuminated by the same pulse is also ploted.

Let us mention that multi-electron ionization and Coulomb explosion of large xenon clusters studied using classical Monte Carlo simulations revealed that electrons are removed from the cluster mainly when the cluster size is enlarged due to Coulomb explosion [13]. Calculation of the energy absorption of atomic clusters as a function of laser pulse length showed a maximum for a critical length which can be linked to an optimal cluster radius [15]. This is with perfect agreement 
with our simulations of argon clusters. Indeed as follows from inspection of Figs. 2 and 3 most effective energy absorption by the free electrons liberated from the atoms but still inside the cluster happens at $\approx 140 \mathrm{fs}$ when the cluster is enlarged approximately twice.

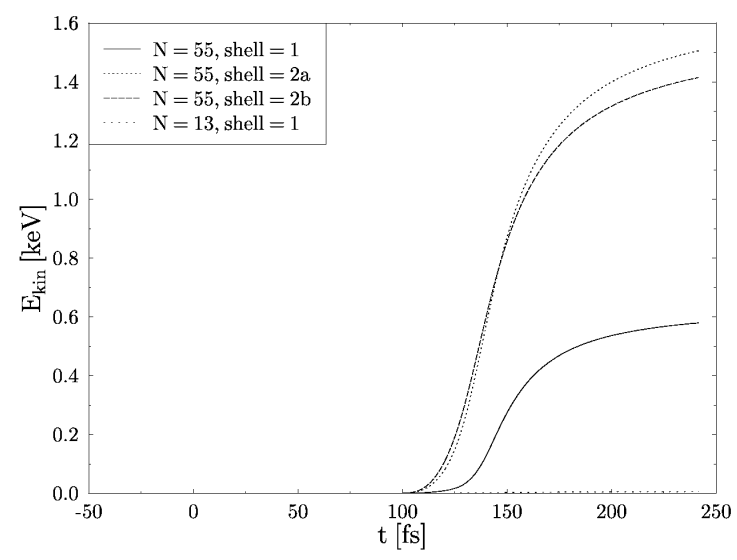

Fig. 4. Average kinetic energy of the outgoing atomic ion fragments coming from different (sub)shells of the 55 atom cluster. Stepwise character of the explosion is seen: the ions leaving first are far more energetic than those leaving later. For comparison average kinetic energy of the ions coming from a 13 atom cluster illuminated by the same pulse is also ploted.

In Fig. 4 we have the kinetic energy $\mathcal{E}_{\text {kin }}$ of the atomic ions fragments versus time $t$. Again it is averaged over the different (sub)shells. Let us note once more the stepwise character of the explosion: the ions leaving first are far more energetic than those leaving later. Such a process has been suggested as an explanation of phenomena observed in experiments [8] and confirmed within 1D numerical simulations using a similar time-dependent Thomas-Fermi hydrodynamic model [21].

For comparison a radius of a 13 atom cluster illuminated by the same pulse is also ploted. We see a strong increase in energy of the ion fragments (coming from an increase in ionization rate) with the number of atoms in the cluster. This is in agreement with TDFT simulations [16].

In Fig. 5 we have average charge of the outgoing atomic ion fragments coming from different (sub)shells of the 55 atom cluster. Field ionization induced by an external laser field starts at the same time for all atoms of the cluster. Next the ionization of the inner shell seems to be slowed down by the presence of the outer shells of the cluster. At later times the outer shells explode and ionization of the inner shell is strongly enhanced by electron impact ionization due to the plasmon-like oscillations inside the cluster. This shows the dependence of atomic ionization rates on the original position of an atom in the cluster and is in agreement with 


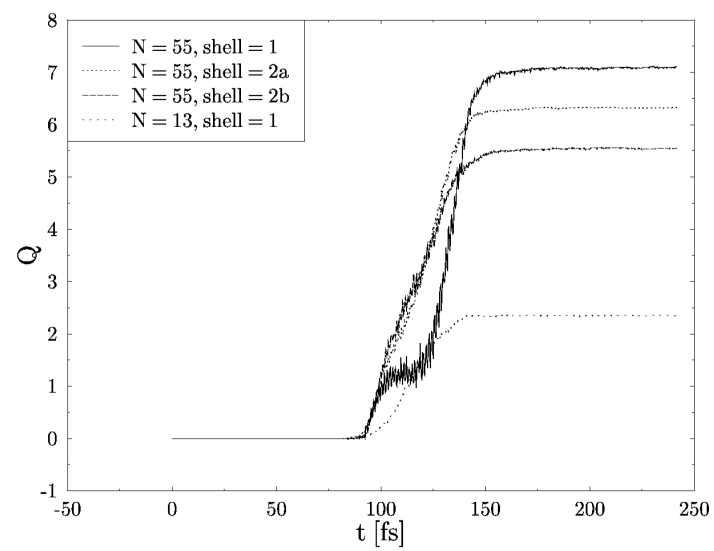

Fig. 5. Average charge of the outgoing atomic ion fragments coming from different (sub)shells of the 55 atom cluster. Field ionization induced by an external laser field starts at the same time for all atoms. At later times ionization of the inner shell is enhanced by electron impact ionization. For comparison average charge of the ions coming from a 13 atom cluster illuminated by the same pulse is also ploted. The ionization rate is much lower.

results of the 1D TDFT simulations [16] (also in our case the most ionized atoms come from the inner shells of the cluster).

For comparison average charge of the ions coming from a 13 atom cluster illuminated by the same pulse is also ploted. The ionization rate is much lower. This is consistent with Fig. 4.

In Fig. 6 we plot the distribution of the final charges of the ions formed in the explosion of a 55 atom cluster. Charges as high as $8^{+}$are observed. The

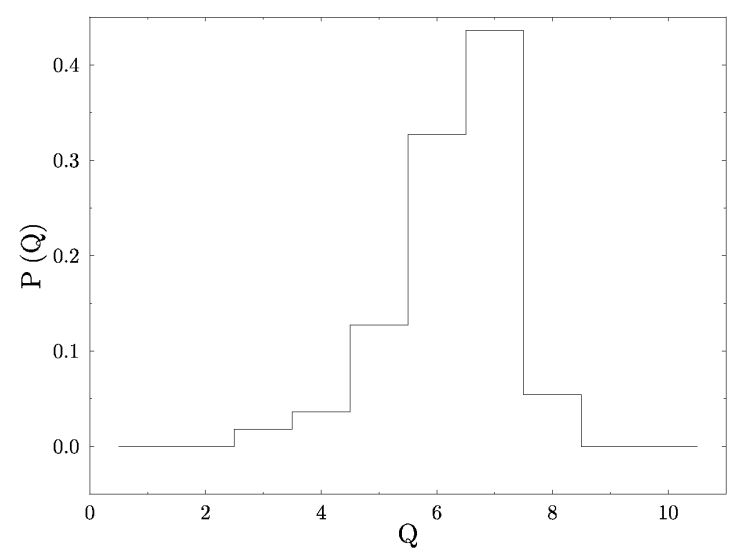

Fig. 6. Distribution of the final charges of the ions formed in the explosion of a 55 atom cluster. They agree with both experimental results and recent theoretical predictions. 
results are consistent with both experimental results by Purnell [22] and theoretical predictions by Ishikawa [12]. It is seen from inspection of Fig. 6 that it is not possible to tell from which shell of the cluster an ion is coming just by looking at its charge. What we need is both charge and kinetic energy.

Thus in Fig. 7 we have the kinetic energy of the ions ploted as a function of the charge state. Small dots correspond to individual ions while larger filled circles represent results averaged over (sub)shells. The linear dependence of the

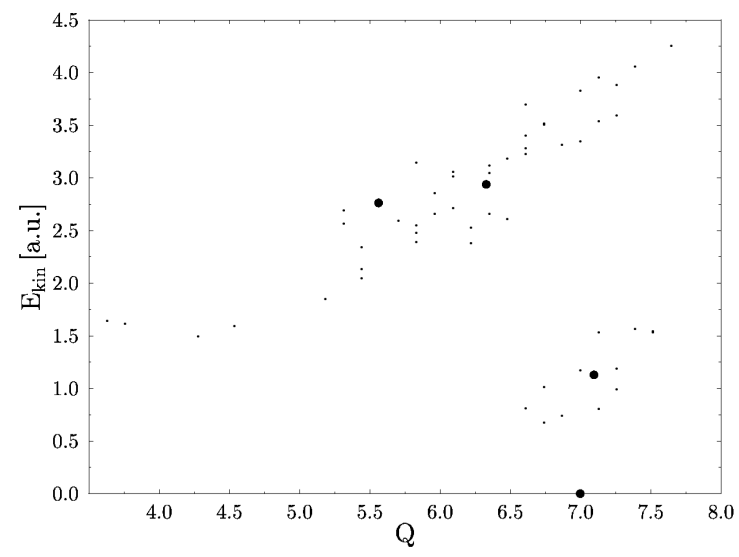

Fig. 7. Kinetic energy of the ions ploted as a function of the charge state. Small dots correspond to individual ions while larger filled circles represent results averaged over (sub)shells. The linear dependence of the ion energy on ion charge seems to be a good approximation for each (sub)shell.

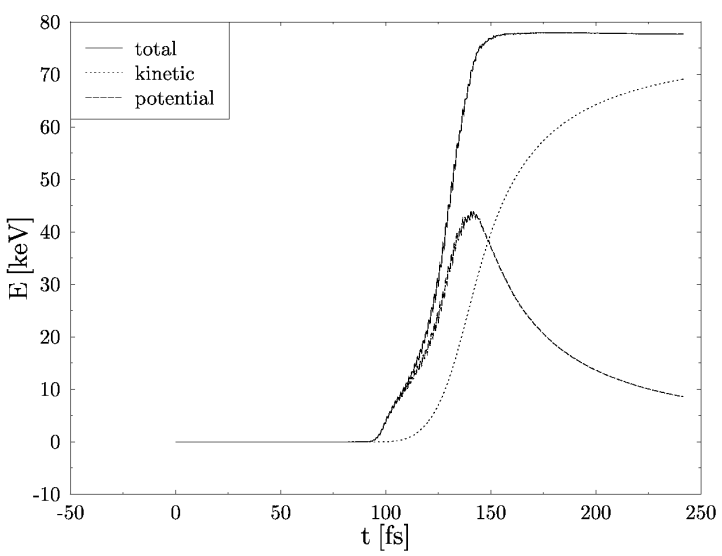

Fig. 8. Kinetic, potential (Coulomb), and total energy of the ions formed in the explosion of the 55 atom cluster ploted versus time. The total energy is constant at the end of the pulse and allows us to estimate the final kinetic energy of the ions. 
ion energy on ion charge seems to be a good approximation for each (sub)shell. This agrees with the theoretical results by Ishikawa [12].

Last but not least in Fig. 8 we present kinetic, potential (Coulomb), and total energy of the ions formed in the explosion of the 55 atom cluster ploted versus time. The total energy is constant at the end of the pulse and allows us to estimate the final kinetic energy of the ions. The final energy of $80 \mathrm{keV}$ agrees with experimental results [22].

\section{Summary}

In summary, hydrodynamic simulations of the interaction of intense, femtosecond, infrared laser pulses with medium-sized argon clusters have been presented. It was shown that for argon clusters considered, the Coulomb explosion is the key process in the explosion mechanism. The dynamics of the outgoing ions appears to be governed purely by the Coulomb repulsion. The physical scenario for the cluster explosion which emerges from our calculations can be divided into three stages: (i) excitation of bound electrons by the laser pulse followed by the onset of ionization and expansion of the cluster; (ii) cluster reaches a certain critical radius for which the laser energy absorption by free electrons inside the cluster is particularly favored, which leads to the production of highly charged ions; (iii) the Coulomb expansion of the remaining ion fragments. Our theoretical model provides a uniform description and consistent explanation of all of the above mentioned stages. Finally, our calculations are in agreement with experimental measurements of the destruction of argon clusters caused by ionization due to an intense femtosecond laser pulse.

\section{Acknowledgment}

This work was supported by the State Committee for Scientific Research under grant No. 5 P03B 06320.

\section{References}

[1] T. Ditmire, T. Donnelly, A.M. Rubenchik, R.W. Falcone, M.D. Perry, Phys. Rev. A 53, 3379 (1996).

[2] A. McPherson, B.D. Thompson, A.B. Borisov, K. Boyer, C.K. Rhodes, Nature 370, 631 (1994).

[3] A. McPherson, T.S. Luk, B.D. Thompson, A.B. Borisov, O.B. Shiryaev, X. Chen, K. Boyer, C.K. Rhodes, Phys. Rev. Lett. 72, 1810 (1994).

[4] T. Ditmire, T. Donnelly, R.W. Falcone, M.D. Perry, Phys. Rev. Lett. 75, 3122 (1995).

[5] E.M. Snyder, S.A. Buzza, A.W. Castleman Jr., Phys. Rev. Lett. 77, 3347 (1996). 
[6] M. Lezius, S. Dobosz, D. Normand, M. Schmidt, J. Phys. B 30, L251 (1997).

[7] T. Ditmire, J.W.G. Tisch, E. Springate, M.B. Mason, N. Hay, R.A. Smith, J. Marangos, M.H.R. Hutchinson, Nature 386, 54 (1997).

[8] M. Lezius, S. Dobosz, D. Normand, M. Schmidt, Phys. Rev. Lett. 80, 261 (1998).

[9] Y.L. Shao, T. Ditmire, J.W.G. Tisch, E. Springate, J.P. Marangos, M.H.R. Hutchinson, Phys. Rev. Lett. 77, 3343 (1996).

[10] T. Ditmire, J.W.G. Tisch, E. Springate, M.B. Mason, N. Hay, J.P. Marangos, M.H.R. Hutchinson, Phys. Rev. Lett. 78, 2732 (1997).

[11] T. Ditmire, Phys. Rev. A 57, R4094 (1998).

[12] K. Ishikawa, T. Blenski, Phys. Rev. A 62, 063204 (2000).

[13] I. Last, J. Jortner, Phys. Rev. A 62, 013201 (2000).

[14] I. Last, J. Jortner, Phys. Rev. Lett. 87, 033401 (2001).

[15] C. Siedschlag, J.M. Rost, Phys. Rev. Lett. 89, 173401 (2002).

[16] V. Véniard, R. Taïeb, A. Maquet, Phys. Rev. A 65, 013202 (2001).

[17] M. Rusek, H. Lagadec, T. Blenski, Phys. Rev. A 63, 013203 (2001).

[18] F. Bloch, Z. Phys. 81, 363 (1933).

[19] K. Huang, Statistical Mechanics, Wiley, New York 1963.

[20] N.H. March, Theory of the Inhomogeneous Electron Gas, Plenum Press, New York 1983, p. 1.

[21] M. Brewczyk, Ch.W. Clark, M. Lewenstein, K. Rzążewski, Phys. Rev. Lett. 80, 1857 (1998).

[22] J. Purnell, E.M. Snyder, S. Wei, A.W. Castelman, Chem. Phys. Lett. 229, 333 (1994). 